\title{
Comparison between local and regional anesthesia in arteriovenous fistula creation
}

\author{
Attilio Ignazio Lo Monte', Giuseppe Damiano ${ }^{1}$, Antonino Mularo' ${ }^{2}$, Vincenzo Davide Palumbo', \\ Rosi Alessi ${ }^{2}$, Maria Concetta Gioviale ${ }^{1}$, Gabriele Spinelli', Giuseppe Buscemi ${ }^{1}$ \\ ${ }^{1}$ Department of Oncological and Surgical Disciplines, Università degli Studi di Palermo - Italy \\ ${ }^{2}$ Department of Biopathology and Forensic Medical Biotechnology, Università degli Studi di Palermo - Italy
}

\begin{abstract}
Purpose: Assessment of the effectiveness of Brachial Plexus Block (BPB) via axillary approach compared to regional anesthesia for arteriovenous fistula surgery in patients affected by end-stage renal disease.

Methods: We compared forty patients randomly divided into two groups. Group A underwent BPB procedure with $15 \mathrm{~mL}$ ropivacaine $1 \%$ and $10 \mathrm{~mL}$ of saline $(0.9 \% \mathrm{NaCl})$ via axillary approach. Group B received local anesthesia with lidocaine $2 \%$. The forearm blood vessels were assessed by Doppler ultrasonography before and after the intervention.

Results: BPB performed on Group A was associated with a considerable venous dilation and a significant decrease (48.7\%, $\mathrm{P}<.05)$ in pulsatility index (PI) measured by Doppler ultrasound. In Group B, PI and venous dilation remained unaltered in the postoperative phase. No complications such as thrombosis or occlusion were encountered among patients who underwent BPB.

Conclusions: The axillary-approached BPB was more advantageous than local anesthesia. Its effectiveness was because of venous dilation and the decrease in the PI, consequent to the reduction in peripheral resistances and the increase in local blood flow, thus offering an ideal background for fistula creation and short-term patency.
\end{abstract}

Key words: Arteriovenous access surgery, Brachial plexus block, Doppler ultrasound, Vasodilation

Accepted: July 9, 2011

\section{INTRODUCTION}

The Registry of the Italian Society of Nephrology shows that, at present, the number of new dialysis patients is $8182(151 /$ million of inhabitants) (1). Diabetic patients represent $12.15 \%$ of the total dialysis population in Italy (2). The creation of arteriovenous fistula is a crucial element for the survival of patients who undergo hemodialysis in order to restore normal equilibrium among body fluids (3). A vascular access is required to allow the "artificial kidney" to drain the blood, and the depurative treatment strictly depends upon the correct functioning of the access. In 1962, Cimino and Brescia performed the first autogenous fistula in the forearm between the radial artery and the cephalic vein $(4,5)$. This started the uninterrupted evolution that has led to today's polytetrafluoroethylene prosthesis. Autogenous access is to be preferred to prosthetic access, the use of prosthesis only being recommended when the peripheral vascular bed is insufficient or exhausted (6). A good vascular access is essential to manage long-term hemodialytic treatment, and it is also important to guarantee a blood flow rate of at least 200$300 \mathrm{~mL} / \mathrm{min}$, avoiding an excessive shunt that may cause heart failure or a vascular steal syndrome.

\section{MATERIAL.S AND METHODS}

From January 2008 to February 2009, forty patients underwent the creation of arteriovenous fistula (AVF) in the forearm between the radial artery and the cephalic vein. The patients enrolled in our study were carefully selected and homogeneously distributed according to age, sex, and concomitant pathologies, and therefore randomized into two groups. All patients signed a written informed consent before the operation. Among our patients, 23 were women $(57.5 \%)$ and 17 were men $(42.5 \%)$. Patients' average age was 66, ranging from 55 to 77 . The pathologies that had led to end-stage renal disease were: diabetes in 15 cases (37.5\%); high blood pressure in 13 cases $(32.5 \%)$; systemic lupus erythematosus (SLE) in 5 cases (12.5\%); glomerulonephritis in 4 cases $(10 \%)$; autoimmune vasculitis in 3 cases $(7.5 \%)($ Tab. I). For patients in the first group (Group A) brachial plexus block (BPB) was performed via axillary approach, while second group patients (Group B) were administered local infiltration with anesthetic. The morning before the operation, all patients were pretreated with intravenous midazolam. They were monitored with ECG, pulse-oximetry and noninvasive blood 
pressure measurement. Patients' forearm blood vessels were measured with Doppler ultrasonography before and after the creation of the AVF. The internal diameter of the basilic vein was measured at the mid-arm antecubital fossa, while the cephalic vein was measured at the wrist. In addition, the diameter of the basilic vein was carefully measured at its entry in the lower axilla. The brachial arterial diameter was also measured, and the blood-flow pattern was recorded using peak systolic velocity and the pulsatility index (PI). The PI is calculated by dividing the difference between maximum and minimum velocity of arterial flow by the mean maximum velocity (7). The surgeon elaborated the best possible procedure for each patient, based on the aforementioned measurements. All of the above represented the pre-anesthetic valuation at time $0\left(t_{0}\right)$.

Group A, comprised of 12 women $(60 \%)$ and 8 men (40\%) (Tab. I), underwent BPB via axillary approach (8). Patients were placed in dorsal position, with their arms abducted to $90^{\circ}$, and their elbows in extension. Once the reference point was identified, patients were administered a cutaneous infiltration with lidocaine $2 \%$. The needle of the electrical stimulator was then inserted and advanced until it caused a motor response in the group of muscles innervated by the radial and median nerves. At first, the current intensity used was high $(2-3 \mathrm{~mA})$, and then gradually reduced near the nervous trunk. Once the nervous fibers were identified, an injection of $15 \mathrm{~mL}$ of ropivacaine $1 \%$ and $10 \mathrm{~mL}$ of saline was administered, for a total volume of $25 \mathrm{~mL}$.

Group B consisted of 11 women (55\%) and 9 men $(45 \%)$ (Tab. I), who underwent local anesthesia in the forearm with lidocaine $2 \%$. Infiltrations were administered by the surgeon.

The arterial and venous vascular assessment was repeated after 5 and 10 minutes for both groups, and the Pulsatility Index Ratio was calculated. The Pulsatility Index Ratio is the ratio between the PI 10 minutes after the block and the PI before the block $\left(\mathrm{PI}-\mathrm{t}_{10} / \mathrm{PI}\right.$ $t_{0}$ ): it represents the magnitude of the sympathetic effect of the block as expressed by the magnitude of the change in the PI.

Data were analyzed using Independent Samples $t$ test. Results are presented as mean values.

\section{RESULTS}

Arterial effects: Group A revealed a significant drop in the PI from 6.53 (range 3.20 to 10.58) before the block, to 4.38 (range 2.20 to 8.85 ) at five minutes, and 3.35 (range 1.20 to 7.85 ) 10 minutes after the block commenced $(P<.05)$, with a $48.7 \%$ total decrease (see Tab. II). The mean Pl Ratio was 0.51 (range 0.02 to 0.76 ). On the contrary, in group B the PI was 5.68 (range 2.15 to $11.45)$ prior to the local anesthesia and remained almost unaltered thereafter: in fact, it was 5.00 at five minutes and 5.23 at 10 minutes from anesthesia. The PI ratio was 0.91 (Tab. II). Data comparing the two groups are shown graphically in Figure 1.

Venous effects: Among group A patients who underwent $\mathrm{BPB}$, venous diameter revealed an increment: basilic vein diameter was $6.40 \mathrm{~mm}$ (range 3.14 to 10.37) before the block, $7.81 \mathrm{~mm}$ (range 3.72 to 12.95) at five minutes, and $8.68 \mathrm{~mm}$ (range 3.86 to 13.72 ) ten minutes after the block commenced $(P<.05)$, with a $26 \%$ average venodilation (Tab. II).

In Group B patients the result was disappointing: cephalic vein diameter was $6.32 \mathrm{~mm}$ (range 2.39 to 12.74) prior to local anesthesia, and $6.60 \mathrm{~mm}$ (range 2.59 to 13.58 ) ten minutes later. A visual comparison

TABLE I - DEMOGRAPHIC DATA OF PATIENTS (DATA EXPRESSED AS MEAN VALUE \pm SD)

\begin{tabular}{lccc}
\hline & Group A * & Group B ** & P \\
\hline Number of patients & 20 & 20 & $11 / 9$ \\
M/F & $12 / 8$ & $66 \pm 7.49$ & 0.5673 \\
Average age and SD & $66.15 \pm 7.55$ & 8 & 0.95 \\
& Primary causes of ESRD & 6 & 0.5592 \\
Diabetes & 7 & 3 & 0.5461 \\
High blood pressure & 7 & 2 & 0.3928 \\
Systemic lupus erythematosus & 2 & 1 & 1 \\
Glomerulonephritis & 2 & & 0.2828 \\
Autoimmune vasculitis & 2 & &
\end{tabular}

* Brachial plexus block group

** Local anesthesia group

ESRD, end-stage renal disease; $F$; female; $M$, male; SD, standard deviation 


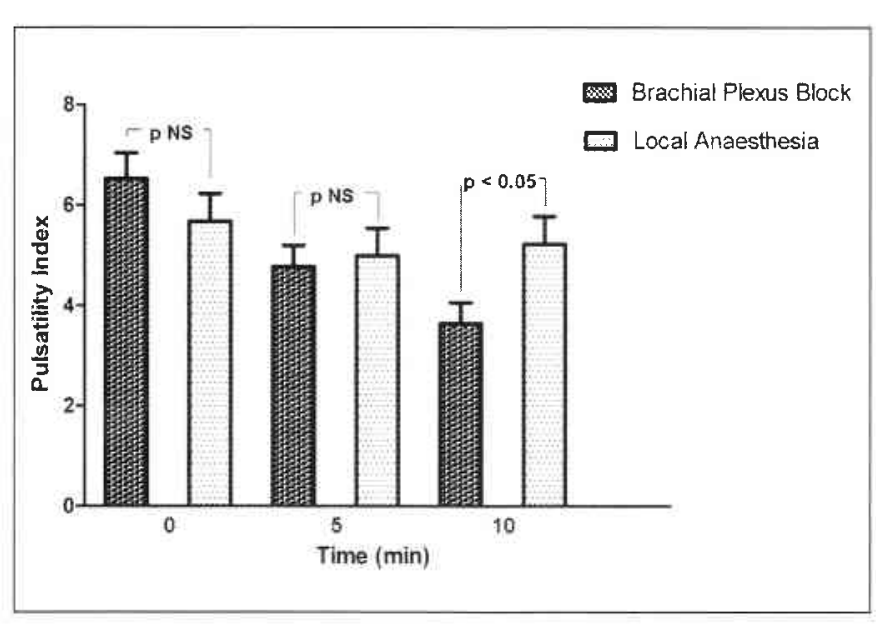

Fig. 1 - Pulsatility Index at 0,5, and 10 minutes following anesthesia during Brachial Plexus Block and Local Anesthesia.

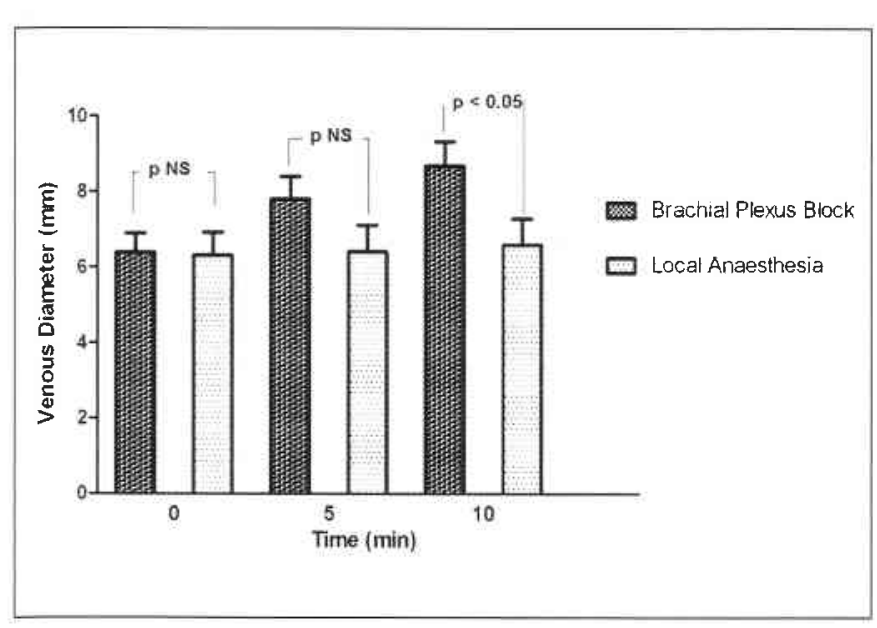

Fig. 2 - Venous diameter at 0,5 , and 10 minutes following anesthesia during Brachial Plexus Block and Local Anesthesia.

TABLE II - RESULTS OF ARTERIAL AND VENOUS EFFECTS BY COMPARING BRACHIAL PLEXUS BLOCK GROUP (A) AND LOCAL ANESTHESIA GROUP (B)

\begin{tabular}{lccc}
\hline Results & Group A * & Group B** & P \\
\hline Arterial effects & & & 0.266 \\
Pl at 0 min & $6.53 \pm 2.3$ & $5.68 \pm 2.43$ & 0.746 \\
Pl at 5 min & $4.38 \pm 1.99$ & $5 \pm 2.43$ & 0.026 \\
Pl at 10 min & $3.35 \pm 1.91$ & $5.23 \pm 2.44$ & $<.9001$ \\
Pl Ratio & $0.51 \pm 0.17$ & $0.91 \pm 0.09$ & $<.45 \pm 8.71$ \\
Decrement of PI & $49.35 \pm 16.99$ & & 0001 \\
Venous effects & & $6.32 \pm 2.7$ & 0.924 \\
Vein diameter before & $6.4 \pm 2.25$ & $6.42 \pm 3.12$ & .1430 \\
Vein diameter at 5 min & $7.81 \pm 2.71$ & $6.6 \pm 3.08$ & 0.035 \\
Vein diameter at 10 min & $8.68 \pm 2.95$ & $4 / 20$ & \\
Complications & $0 / 20$ & & \\
\hline
\end{tabular}

\footnotetext{
* Brachial plexus block group

** Local anesthesia group

$\mathrm{PI}$, Pulsatility Index; PI Ratio, Pulsatility Index Ratio $=\mathrm{PI}_{\mathrm{Tromin}}-\mathrm{PI}_{\mathrm{TO}}$
}

of venous diameter at 0,5 , and 10 minutes after anesthesia in brachial plexus block and local anesthesia is presented in Figure 2.

Complications: We considered the possible onset of complications such as early thrombosis within the first week from the operation. However, during this experience, we did not encounter thrombosis cases among Group A patients, but four Group B patients who underwent local anesthesia developed early thrombosis, and for these patients it was necessary to perform a new intervention.

\section{DISCUSSION}

Brachial plexus normally guarantees the innervation of almost the whole upper arm. Radial, ulnar, and musculocutaneous nerves are separated from the vascular-nervous bundle at the axillary fossa level (9); for this reason the block must be practiced at the highest point of the axilla. The block through this approach enables obtaining a good anesthetic effect for surgeries distal to the elbow and produces a low risk of involving the surrounding structures since the anesthetic is injected at a suitable distance from the vascular-nervous bundle; moreover, it reduces the oc- 
currence of pneumothorax. Unfortunately, this method does not involve the musculocutaneous nerve which leaves the vascular-nervous bundle near the axillary fossa. Therefore, the volume of the anesthetic is more important than its concentration because it involves a larger number of nervous fibers. Identification of the nervous roots by electric stimulation enables an analgesia area from elbow to hand. It is fundamental to ascertain the technique to carry out the block, the reference points, the anatomic structures, and the direction of the needle. Some operators prefer to perform the axillary block by paresthetic or transarterial technique. However, our experience suggests that these approaches are burdened by a higher occurrence of complications during the execution of techniques such as neurologic or vascular complications. Neurologic complications may be consequent to direct nerve trauma, hematoma formation or edema with subsequent ischemia from compression of the nerve, intraneural injection, as well as direct toxicity of anesthetic agent; their occurrence may lead to temporary or permanent post-operative paresthesias and paralysis.

The vascular complications that may occur during execution of axillary block are represented from accidental intravascular injection, arterial occlusion (because of compression by hematoma or tourniquet), and formation of hematoma (because of mechanical injury at the level of the axillary vessels).

The aim of neurostimulation is to localize the nerve avoiding direct contact with its fibers, thus reducing the risk of direct traumatic lesions. Using a very low-intensity electric current, the needle tip will need to be placed too close to the nerve in order to obtain muscle contractions, thus risking damage to the nerve. Instead, if the intensity of the current is too high, contractions will appear when the needle tip is still far from the neurovascular sheath, thus risking injecting all or part of the anesthetic solution outside the area of interest.

BPB determines a vasoplegic effect that causes a venous dilation and a decrease in the pulsatility index, optimizing the creation of the primary AVF, especially in patients with a reduced or exhausted superficial vascular bed. Such positive effects are not present when the arteriovenous fistula is performed by infiltration of local anesthetic into the area of interest (10).

Arterial and venous dilation are crucial elements in determining the destiny of an arteriovenous fistula because they immediately guarantee sufficiently high blood flow to prevent premature failure of the fistula itself. These effects can be maintained for various hours during the post-operative period when BPB is made by single shot; however, their duration can be extended further if the block procedure is performed by using a catheter in the brachial neurovascular compartment.

During the post-operative period it was observed that the mean PI remained rather low: it was calculated as equal to 2.45 at 15 minutes from performing the AVF and it maintained its value of 3.00 until the fifth hour following the operation. This low average PI value has probably contributed to maturation of the fistula with the consequent arterialization of the venous wall and reduction in the risk of early thrombotic occlusion.

Patients were assessed for 100 days after the operation, and during that period two early failures were recorded in group $B$ patients. No thrombotic complications occurred in group A patients.

Four cases of thrombosis occurred in Group B which we hypothesized were because of the reduced compliance of the venous tract in the first few hours following the operation. In fact, no other predictable factors could be considered responsible for early thrombosis.

Brachial plexus block via axillary approach is more advantageous than local anesthesia. The greater effectiveness is given by the venous dilation and the reduction in the PI. This determines a decrease in peripheral resistances and an increase in local blood flow (offering an ideal background for end-stage renal disease patients whose survival depends upon arteriovenous fistula) thanks to the sympathectomy-like effects of the block that we quantified using the PI Ratio. The PI Ratio is obtained by dividing the $\mathrm{PI}$ value 10 minutes from administering anesthesia by the PI value found at $t_{0}$ prior to the block. If the PI Ratio is less than 1 , it means that there is a sympathectomy-like effect expressed by the increase in diastolic flow and the reduction in the peripheral resistances. The greater the fall in the $\mathrm{PI}$ ratio, the larger the sympathectomy-like effect. Such effect can facilitate better use of the patrimony of autogenous veins.

The results obtained seem to show a reduced risk of early complications, such as occlusion and thrombosis, in arteriovenous fistula. On the contrary, according to our data, these complications occur more frequently in patients who undergo the process of arteriovenous access under local anesthesia (11). The incidence of complications related to the use of this peripheral block technique is minimized by using neurostimulation in the hands of a trained operator with sufficient experience. The venous dilation which occurs with BPB, probably determines a minor resistance to the shunted flow in the early phases following creation of the fistula; in this way, compliance of the venous bed persists for a vascular tract length and sufficient time so as to permit the arteriovenous fistula to maintain spontaneously. The aspects related to the comparison of the maturation time between the two groups have not been well investigated, since the first use of the AVF strictly depends on the clinical condition of patients (grade of renal failure). By convention, it is preferable to wait about 30 days before using the AVF in order to avoid late thrombosis and occlusion consequent to a AVF that is not well matured.

This study highlights the importance of integration 
among different medical specialties in the cure of patients, offering the best strategy in order to manage complex problems of modern medicine and to guarantee the best possible quality of life for hemodialysis-dependent patients.

Financial support: None.

Conflict of interest: None to declare.
Address for correspondence

Attilio Ignazio Lo Monte

Associate Professor of General Surgery

Facoltà di Medicina e Chirurgia, Università degli Studi di Palermo Dipartimento di Discipline Chirurgiche ed Oncologiche, Università degli Studi di Palermo

Via del Vespro 129

90127 Palermo, Italy

ailomonte@unipa.it

\section{REFERENCES}

1. Alloatti S, Quarello F, Salomone M et al. Census 2004 of the Italian Renal and Dialysis Units. G Ital Nefrol. 2007;24:141150.

2. Panzetta G, Basile C, Santoro A et al. Diabetics on dialysis in Italy: a nationwide epidemiological study. Nephrol Dial Transplant 2008;23:3988-3995.

3. Sidawy Anton N, Spergel Lawrence M et al. The Society for Vascular Surgery: clinical practice guidelines for the surgical placement and maintenance of arteriovenous hemodialysis. IVasc Surg. 2008;48:2S-25S.

4. Cimino JE, Brescia J. Simple venipuncture for hemodialysis. $N$ Engl J Med. 1962;267:608-609.

5. Cimino JE, Brescia JB, Appel $\mathrm{K}$ et al. Chronic hemodialysis using venipuncture and a surgically created arteriovenous fistula. N Engl J Med. 1966;275:1089-1092.
6. Santoro A, Canova C, Freyrie A et al. Vascular access for hemodialysis. J Nephrol. 2006;19(3):259-264.

7. Shemesh D, Olsha O, Orkin D et al. Sympathectomy-like effect of brachial plexus block in arteriovenous access surgery. Ultrasound Med and Biol. 2006;32:817-822.

8. Deleuze A, Gentili M-E. Regional block for the elbow, wrist and hand. Ann Fr Anesth Reanim. 2006:25:242-248.

9. De Jong RH. Axillary block of the brachial plexus. Anesthesiology 1961:22:215-225.

10. Laskowski IA, Muhs B, Rockman CR et al. Regional nerve block allows for optimization of planning in the creation of arteriovenous access for hemodialysis by improving superficial venous dilatation. Ann Vasc Surg. 2007;21:730733.

11. Ravani P, Marcelli D, Pecchini P et al. Early failure rates of arteriovenous fistulas for haemodialysis: evaluation of sixyear activity. IVasc Access 2001;2:154-160. 